\title{
Three-dimensional Investigation of Retention Time Distribution of Waste Stabilisation Ponds
}

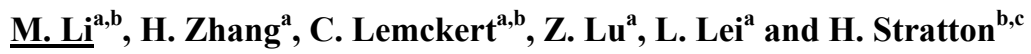 \\ ${ }^{a}$ Griffith School of Engineering, Griffith University, Gold Coast Campus, QLD 4222, Australia \\ ${ }^{b}$ Smart Water Research Centre, Griffith University, Gold Coast Campus, QLD 4222, Australia \\ ${ }^{c}$ School of Biomolecular and Physical Sciences, Griffith University, Nathan Campus, QLD 4111, Australia \\ Email: $\underline{\text { m.li@griffith.edu.au }}$
}

\begin{abstract}
Waste stabilisation ponds (WSPs) use natural microbiological, photosynthetic, biochemical, physico-chemical and hydrodynamic processes to treat wastewater. They require little technical attention during operation and are less demanding in terms of construction cost and energy consumption than other engineered wastewater treatment systems. Practical engineering experience and research over the past few decades have established that hydrodynamics is crucial in determining the treatment efficiency of WSPs. After reviewing a large number of pond systems operated in Australia, Wood et al. (1995) stated that many systems were found to operate below an optimal level due to a reduced retention time of wastewater. It is therefore required that the hydrodynamic retention time distribution be precisely understood so that the hydrodynamic behaviour and the overall treatment efficiency of WSPs can be accurately evaluated.
\end{abstract}

A substantial number of models have been developed to look into various hydrodynamic aspects of WSPs. However, most of the work has been limited to one or two dimensions due to computational capabilities. The present study aims to establish a generic model fully describing the three-dimensional hydrodynamic behaviour of WSPs. MIKE by DHI is chosen as the modelling tool considering the following favourable features: (1) applying a non-hydrostatic engine to simulate unsteady three-dimensional flows; (2) taking into account density variation, bathymetry and external forces such as meteorology, tidal events and currents; (3) incorporating several modules such as hydrodynamic, transport and ecological, which allows integrated modelling of hydrodynamic and biological processes for a more complete analysis and prediction of wastewater treatment.

A typical pond model with the dimensions of $50 \mathrm{~m}$ (length) by $20 \mathrm{~m}$ (width) by $1.5 \mathrm{~m}$ (depth) was adopted. The model was validated against an empirical formula for the wind-driven circulation in a tank. An optimised meshing scheme of $1 \mathrm{~m}$ by $1 \mathrm{~m}$ horizontally and 7 layers vertically was determined to achieve the best computational performance-time ratio. Subsequently, the validated model was employed to formulate WSP retention time analysis.

A parametric study was conducted in terms of varying length to width ratio $(L / W)$, inlet/outlet positioning, inlet direction changes, varying wind speed and direction. It was found that:

- Pond $L / W$ ratio has a significant influence on pond retention time. It was noted that a larger $L / W$ ratio is associated with longer retention time.

- Winds are a predominant factor in WSP performance, especially wind direction. The retention time of wastewater was found to be longer due to the circulation in the transverse direction generated in the pond when wind direction was perpendicular to the inflow direction.

- The inlet/outlet position change and the inflow direction variation present a rather mild influence on pond retention time in comparison to pond $L / W$ ratio and winds. This is partially due to the local boundary effect in the modelling. Another possible reason can be associated with the pond configuration specified in this study.

This study performs a systematic investigation of WSP retention time based on three dimensional models. It is expected to establish a modelling framework for extended WSP studies. The parameter analysis presented in this work will be further developed to address the interrelated effects of multiple parameters on WSP hydrodynamics. Ultimately, this study will lead to the development of a 3D model which will incorporate the fate of pathogens linked to hydrodynamic parameters. This model will allow overall efficiency evaluation of the design, operation, retrofit and maintenance of WSPs.

Keywords: $\quad$ Three dimensions, numerical modelling, Waste Stabilisation Ponds, retention time distribution, hydrodynamics 


\section{INTRODUCTION}

Waste stabilisation ponds (WSPs) are widely used for wastewater treatment throughout the world. They rely on microbiological, photosynthetic, biochemical, physico-chemical and hydrodynamic processes to treat wastewater, and require less technical attention compared to mechanical wastewater treatment plants. Studies evaluating the treatment efficiency of WSPs show that hydrodynamics is a crucial element affecting the overall performance of WSPs. Watters et al. (1973) stated that 'the hydrodynamic flow characteristics will have an effect on the dispersion and retention time of the wastewater, and consequently on the efficiency of removing organic and pathogenic organisms'. The Department of Environment and Planning (1992) in the state of Tasmania surveyed 39 wastewater treatment systems and reported that $74 \%$ of the pond systems failed to achieve the discharge requirements, and this was largely due to hydrodynamic problems, namely, reduced retention time of wastewater in the pond. It is of primary importance that the retention time of WSPs and the factors affecting its distribution be clearly understood before WSP treatment efficiency can be accurately evaluated.

A substantial number of models have been developed to look into various hydrodynamic aspects of WSPs. Wood et al. (1995) employed a finite element based CFD software, FIDAP, to solve the governing NavierStokes equations. This study was limited to two dimensions. The inlet and outlet openings were assumed over the entire depth of the pond. Any effects regarding the pond depth were not considered. A further study was conducted by Wood et al. (1998), leading to the conclusion that two-dimensional CFD models were not able to model the three-dimensional inlet behaviour and hence couldn't give an adequate description of WSP hydrodynamics. Consequently, a three-dimensional study was presented in Wood (1997). However, the findings could be considered weak as the model was oversimplified and restricted by computational limitations. Vega et al. (2003) employed a two-dimensional depth-integrated model using MIKE 21 to study the hydrodynamic and transport (advection-dispersion) processes in a full-scale anaerobic pond. The model assumed that vertical velocities are small, thus the vertical acceleration is negligible with respect to the horizontal component. Abbas et al. (2006) investigated how the pond geometry affects the treatment efficiency of WSPs using a two-dimensional depth-integrated model. Kennedy et al. (2006) performed a three-dimensional estimation of the hydraulic retention time of the Wachusett reservoir in Massachusetts. Very recently, Sah et al. (2011) developed a pond-specific integrated three-dimensional model incorporating both hydrodynamics and mechanistic water qualities. However, results from both three dimensional analyses were not completely validated.

It is noted that most previous WSP modelling work has been limited to one or two dimensions. Badrot-Nico et al. (2010) stated: "two dimensional models were ill-adapted to the modelling of WSPs which, despite being shallow water bodies, are subject to influential three-dimensional mechanisms". It is therefore the motivation of this study to establish a generic three-dimensional model to fully describe the hydrodynamic behaviour of WSPs. MIKE by DHI is chosen as the modelling tool considering the following favourable features (Scientific Documentation, MIKE 3 Flow Model, Hydrodynamic Module, 2012): (1) applying a nonhydrostatic engine to simulate unsteady three-dimensional flows; (2) taking into account density variation, bathymetry and external forces such as meteorology, tidal events and currents; (3) incorporating hydrodynamic, transport and ecological sub-modules to account for the physical, chemical and biological processes of WSPs.

The following 5 sections (2-6) presented in this manuscript describe the basis of developing a generic WSP 3D model for determining the fate of pathogens and chemicals. The work thus far includes a mathematical formulation of the physical problem is presented in Section 2; the development of the MIKE 3 model is introduced in Section 3, including the basic model setup, model convergence test and validation; a numerical example is presented in Section 4, illustrating a full evaluation process of WSP hydrodynamics, along with introducing some basic problem settings, flow pattern examination and the interpretation of hydrodynamic retention time of the WSP; and Section 5 presents a parametric analysis on the effect of several factors on WSP retention time. It is followed by Section 6, which concludes the paper by highlighting the main findings of this study and proposing guidelines for future work.

\section{HYDRODYNAMIC FORMULATION OF WSPS}

The mathematical formulation of the flow in a WSP needs to address mass conservation, momentum conservation, conservation of salinity and temperature and the equation of state relating local density to salinity, temperature and pressure. In three dimensions, the governing equations of the flow in a WSP are (Scientific Documentation, MIKE 3 Flow Model, Hydrodynamic Module, 2012): 


$$
\begin{gathered}
\frac{1}{\rho c_{s}^{2}} \frac{\partial P}{\partial t}+\frac{\partial u_{j}}{\partial x_{j}}=S S \\
\frac{\partial u_{i}}{\partial t}+\frac{\partial\left(u_{i} u_{j}\right)}{\partial x_{j}}+2 \Omega_{i j} u_{j}=-\frac{1}{\rho} \frac{\partial P}{\partial x_{i}}+g_{i}+\frac{\partial}{\partial x_{j}}\left(v_{T}\left(\frac{\partial u_{i}}{\partial x_{j}}+\frac{\partial u_{j}}{\partial x_{i}}\right)-\frac{2}{3} \delta_{i j} k\right)+u_{i} S S
\end{gathered}
$$

Equation (1) is the mass equation, in which $\rho$ is the density of the fluid; $c_{s}$ is the speed of sound in water; $u_{j}=$ $u, v, w$ when $j$ varies from 1 to 3 are the velocity components; $x_{j}=x, y, z$ and $t$ are the spatial and temporal variables; $P$ is the fluid pressure; $S S$ refers to the source and sink terms. Equation (2) is the Reynoldsaveraged Navier-Stokes equations, in which $\Omega_{i j}$ represents the Coriolis tensor; $g_{i}$ stands for the gravitational acceleration; $v_{T}$ denotes the turbulent eddy viscosity; $\delta_{i j}$ is the Kronecker's delta $\left(\delta_{i j}=1\right.$ if $i=j$ and $\delta_{i j}=0$ if $i$ $\neq j$ ) and $k$ is the turbulent kinetic energy. In (1) and (2), subscript $j$ is a summation index, meaning that $j$ subscripted terms are to be summed over when $j$ varies from 1 to 3; subscript $i$ is a free index, resulting in three equations in the $x, y$ and $z$ directions respectively when $i$ runs from 1 to 3 .

The transport of salinity $S$ and temperature $T$ in a WSP is captured by advection and dispersion, and it is mathematically described by:

$$
\frac{\partial C}{\partial t}+\frac{\partial}{\partial x_{j}}\left(C u_{j}\right)=\frac{\partial}{\partial x_{j}}\left(D_{j} \frac{\partial C}{\partial x_{j}}\right)+S S
$$

where, $C$ can be replaced by $S$ for salinity and $T$ for temperature. $D_{j}=D_{x}, D_{y}$ and $D_{z}$ are the dispersion coefficients in the $x, y$ and $z$ directions. Equation (3) states that the change in salinity or temperature is equal to the difference between the ingoing and outgoing transport plus the source-sink contribution.

Equations (1) to (3) are to be solved under specific boundary conditions in terms of pressure (or surface elevation) at open boundaries; bathymetry; bed resistance; external forces such as wind and source and sink conditions. The solution of the equations is also subjected to initial conditions about velocity and pressure (or surface elevation).

\section{MODEL DEVELOPMENT}

\subsection{Model setup}

A typical example of a WSP, as shown in Figure 1 (a), can be presented by a rectangular-shaped basin equipped with inlet and outlet pipes as illustrated in Figure 1 (b). $L, W$ and $D$ denote the length, width and the depth of the pond, respectively. The inlet and the outlet are positioned on two side walls $A C$ and $A^{\prime} C^{\prime}$. A Cartesian coordinate system is constructed with its origin seated at point $A$. The $x$ and $y$ axes are in alignment with the longitudinal and the transverse axes of the pond, and the $z$ axis is pointing positively upwards. Horizontally, a structured mesh is applied with rectangular cells uniformly sweeping through the $x y$ plane. The mesh is then projected throughout the entire pond depth into several layers in the vertical direction.

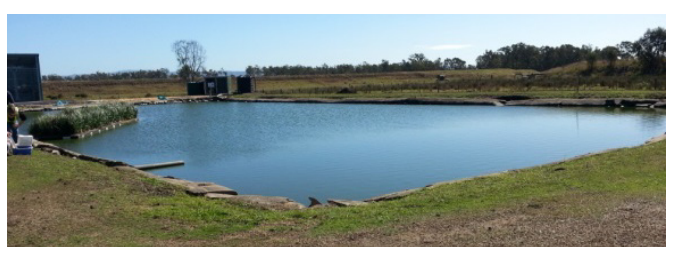

(a)

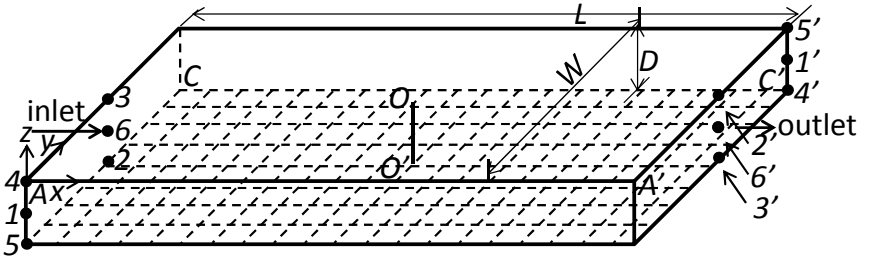

(b)

Figure 1. Illustration of a typical WSP: (a) field WSP prototype and (b) WSP numerical model

\subsection{Convergence test and model verification}

To examine the performance of the model in terms of its convergence behaviour and validity, the wind driven set-up in a pond is chosen as a benchmark case, as the solution of this problem is readily available. Winds with a constant velocity of $5 \mathrm{~m} / \mathrm{s}$ blow over the surface of the pond. The pond is $50 \mathrm{~m}$ in length, $20 \mathrm{~m}$ in width and $1.5 \mathrm{~m}$ in depth. The set-up of the water surface is given in the empirical equation as (User Guide, MIKE 3 Flow Model FM, Hydrodynamic Module, 2012): 


$$
\Delta h=\frac{\tau_{w}}{g \rho} \frac{L}{d}
$$

where $\Delta h$ is the wind set-up and $d$ is the still water depth. The velocity profile of a cross section at some distance from pond side walls $A C$ or $A^{\prime} C^{\prime}$ in Figure 1(b) approximately follows a logarithmic profile, and is given as follows:

$$
\frac{u}{U_{\tau}}=\frac{1}{\kappa}\left(1+\ln \left(\frac{-z}{d}\right)\right)
$$

In (5), $u$ is the $x$ velocity component at any $z$ level, $\kappa=0.41$ is the von Karman constant; $U_{\tau}=\max \left(U_{\tau s}, U_{\tau b}\right)$, with $U_{\tau s}$ and $U_{\tau b}$ being the friction velocities associated with the surface and bottom stresses.

In total, 9 different meshing schemes were tested, aiming at determining the optimal mesh resolution for both horizontal and vertical directions. The wind set-up $\Delta h$ and corresponding relative error $e_{R}$ with respect to the $\Delta h$ calculated from equation (4) are listed in Table 1. The velocity component in the $x$ direction along line $O-O^{\prime}$ (see Figure 1(b)) from 5 chosen cases (for clearer presentation) is compared with equation (5) in Figure 2. In Table 1 and Figure 2, mesh scheme 1-1-5 means the grid size is $1 \mathrm{~m}$ in the $x$ direction, 1 $\mathrm{m}$ in the $y$ direction and 5 water layers in the $z$ direction. It is shown that results from numerical models generally agree well with the empirical formulations, which demonstrates the credibility of the proposed model. Taking into consideration the calculation time involved and the level of accuracy achieved for both the wind set-up and velocity profile, a horizontal grid size of $1 \mathrm{~m}$ by $1 \mathrm{~m}$ with 7 layers in the vertical direction are suggested as the best mesh resolution for subsequent analyses.

\section{NUMERICAL EXPERIMENT}

A typical WSP is studied in this section, using the same pond dimensions as specified in the previous section. The inlet and outlet are located at points 6 and 6' as shown in Figure 1 (b). According to practical engineering data, the inflow rate $Q=0.005 \mathrm{~m}^{3} / \mathrm{s}$; inflow velocity $v=0.01 \mathrm{~m} / \mathrm{s}$. The inflow direction is normal to the side wall $A C$. No winds or thermal effects are considered in this case. A 10-day simulation period with a time interval of $1 s$ is calculated. It is noticed that the model arrives at a steady state approximately 15 minutes after the simulation starts. The steady state data is used for the following result interpretation.

\subsection{Examination of flow property}

The velocity vector field in the $x y$ plane at the middle depth of the pond is shown in Figure 3.

It corresponds to the layer where the inlet and outlet are located, but it does represent the velocity vector distribution pattern for all layers. The velocity around the inlet area is of a bigger magnitude. It becomes
Table 1. Wind set-up calculated from different mesh schemes and equation (4)

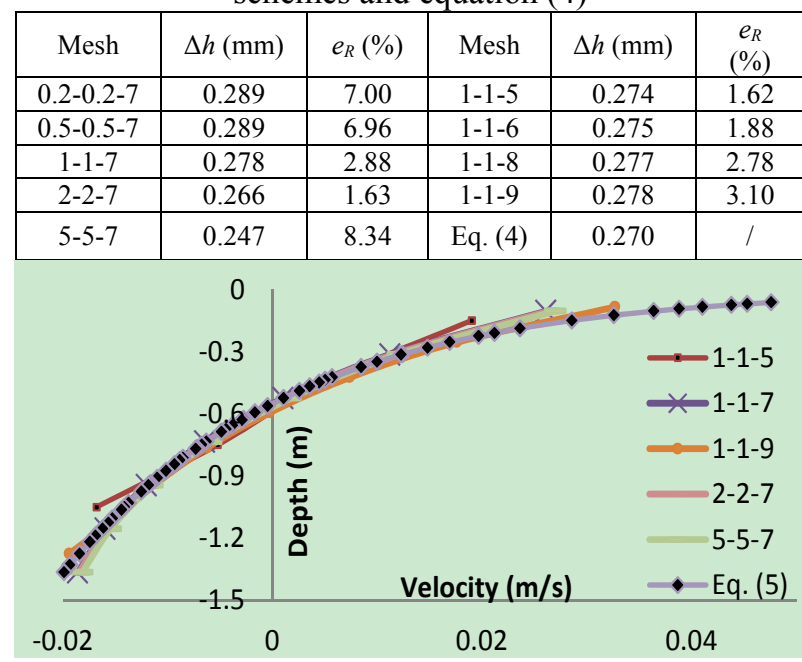

Figure 2. Comparison of velocity profile between different mesh schemes and equation (5)

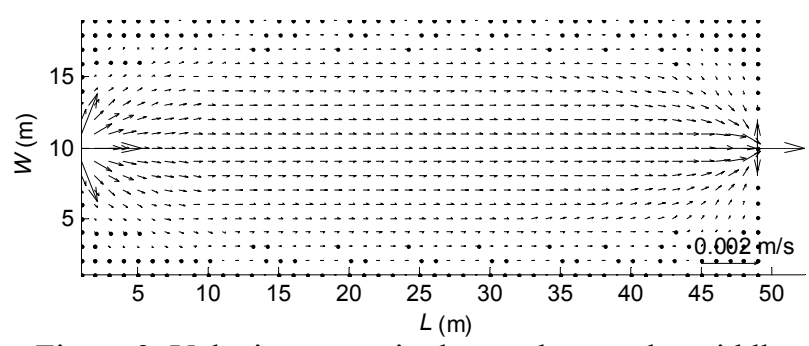

Figure 3 . Velocity vector in the $x y$ plane at the middle

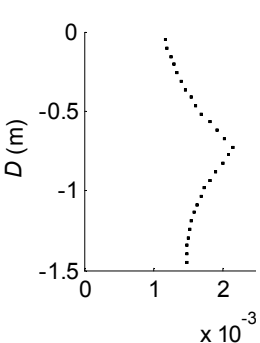

(a)

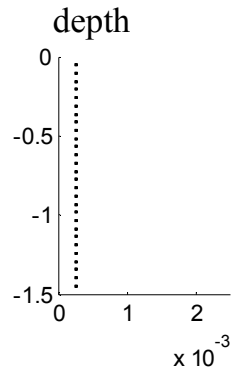

(b)

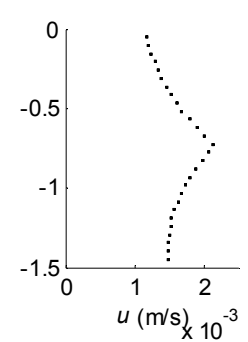

(c)
Figure 4. Velocity profile along pond depth (a) at the inlet; (b) in the middle and (c) at the outlet 
more uniform across the pond longitudinal direction when it comes to the centre of the pond. Moving towards the outlet, the velocity increases again. This feature can also be noticed in Figure 4, in which $u$ velocity profiles along pond depth are plotted at three locations, i.e. close to the inlet (a), at the middle (b) and close to the outlet (c). It is also noted that around the inlet/outlet area, velocity is much greater at the depth where the inlet and outlet are located, whereas those at the top and the bottom of the pond are much smaller due to the effect of shear stresses. The calculation of the Reynolds number of the flow in the pond leads to a value of 200, which indicates that in this situation, the flow is laminar flow. This will vary when external forces are imposed on the flow, especially winds.

\subsection{Hydrodynamic retention time of WSPs}

One of the most informative characterisations of WSPs is the hydraulic retention time. Theoretically, the hydraulic retention time $T_{\text {theor }}$ is defined as the ratio of the pond volume $V$ to the pond inflow rate $Q$. A WSP is expected to exhibit an actual retention time of $T_{\text {theor }}$ to achieve optimised treatment efficiency. However, in practice, WSPs are always found to function below the optimised level due to unevenly distributed flow patterns in three dimensions. To identify reasons for hydraulic deficiencies and thus propose corresponding retrofitting solutions, tracer studies are often used to track the flow path, locations of stagnant space, time of peak outflow, etc. In this model, the advection-dispersion module (equation(3)) is employed to conduct the tracer analysis. A certain amount of simulated tracer was dropped into the pond on the $4^{\text {th }}$ day in the model when the flow in the WSP has been fully developed. By measuring the variation of tracer concentration $C(t)$ at the outlet, a retention time distribution function $E(t)$ was calculated as: $E(t)=C(t) / \int_{0}^{\infty} C(t) d t . E(t)$ quantitatively describes the period of time different water particles have spent in the pond. Another function, termed the cumulative distribution function $F(t)$, is also used to examine WSP's response to a certain inflow condition: $F(t)=\int_{0}^{t} E(t) d t . F(t)$ returns the fraction of tracer particles that have resided in a WSP no longer than a period of time $t$. As illustrated in Figure 5 (b), $80 \%$ tracer particles have resided in the pond for 4 days or less.

Another temporal
parameter measures
the average time
tracer particles
spend in a WSP
before exit. It is
calculated as the
first moment of the
$E(t)$ function, and
termed the mean
retention time $t_{m}$

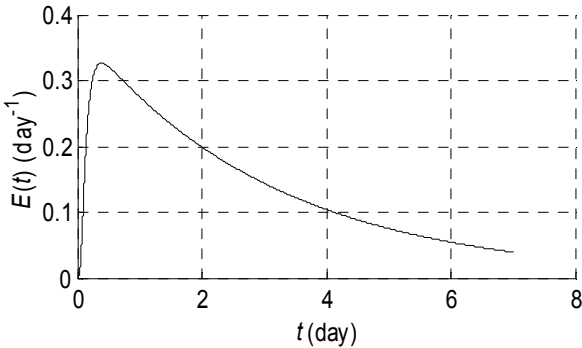

(a)

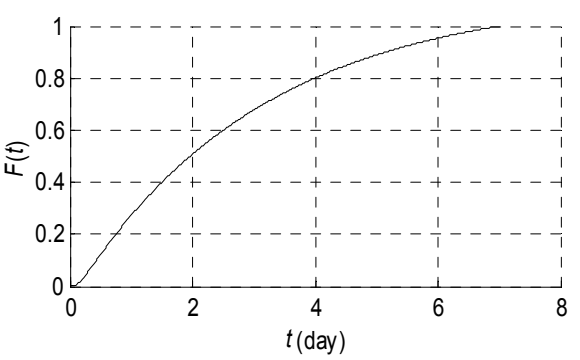

(b)

Figure 5 Tracer analysis for the case study: (a) $E(t)$ function and (b) $F(t)$ function interpreting WSP retention time, such as the time corresponding to the passage of the $16^{\text {th }}$ percentile of the tracer through the outlet $t_{16}$ (Persson, 2000), or the peak flow $t_{p}$. In this example, $t_{16}, t_{p}$ and $t_{m}$ are $0.625 d$, $0.39 d$ and $2.40 d$ for a pond with $T_{\text {theor }}=3.47 d$, respectively.

\section{PARAMETRIC ANALYSIS ON WSP HYDRAULIC EFFICIENCY}

Critical factors such as pond dimensions, inlet/outlet layout, inflow conditions and environmental forces change in real situations, and hence WSPs present varied behaviour. A parametric study was conducted to examine how these parameters affect WSP's retention time. It is organised in terms of varying pond length to width ratio $(L / W)$, inlet/outlet positioning, inlet direction changes and varying wind speed and direction.

\subsection{Effect of $L / W$}

A wide range of $L / W$ from 0.1 to 10 , represented by the six cases shown in Table 2, was examined. The pond was designed to vary only the length and the width and the pond volume was kept constant to ensure an identical $T_{\text {theor }}$ of 3.47 days for all cases. The inlet and the outlet were positioned at point 6 and 6' shown in Figure 1 (b), respectively. An inflow velocity of $0.01 \mathrm{~m} / \mathrm{s}$ and a direction in alignment with the pond inletoutlet direction were enforced. No winds were considered in this scenario. The $E(t)$ and $F(t)$ functions corresponding to three cases $L / W=0.1,1.6$ and 10 are plotted in Figure 6 (a) and (b), respectively. All $E(t)$ 
curves are left-skewed with the time of peak $E(t)$ values increasing from 0.026 days, 0.278 days to 0.993 days. The long tails of all three curves merge after approximately 5 days, as the majority of tracer particles have left the pond within 5 days and the amount of tracer exiting the pond after 5 days is rather insignificant. As shown in Table 2, all temporal parameters $t_{16}, t_{p}$ and $t_{m}$ are greater in cases with larger $L / W$. This is attributed to the fact that the larger the $L / W$ is, the further apart the outlet is from the inlet, meaning water particles have to travel further and hence stay longer in the pond.

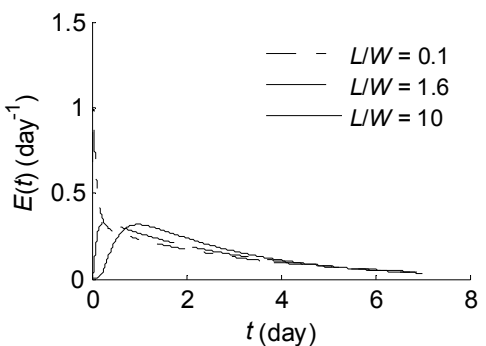

(a)

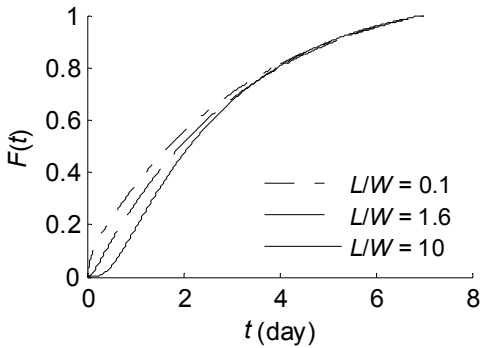

(b)

Figure 6. Tracer analysis of ponds with varying $L / W$ : (a) $E(t)$ function and (b) $F(t)$ function

Table 2. Hydrodynamic retention time measures of ponds with varying $L / W$

\begin{tabular}{|c|c|c|c|c|}
\hline No & $L / W$ & $t_{16}$ & $t_{p}$ & $t_{m}$ \\
\hline 1 & 10 & 0.924 & 0.993 & 2.529 \\
\hline 2 & 2.5 & 0.625 & 0.390 & 2.399 \\
\hline 3 & 1.6 & 0.579 & 0.278 & 2.378 \\
\hline 4 & 0.625 & 0.524 & 0.129 & 2.347 \\
\hline 5 & 0.4 & 0.497 & 0.051 & 2.328 \\
\hline 6 & 0.1 & 0.285 & 0.026 & 2.184 \\
\hline
\end{tabular}

\subsection{Effect of inlet/outlet locations}

The study of the effect of inlet/outlet positioning on the hydraulic retention time of WSPs was designed as explained in Figure 1(b). A total of 6 different inlet/outlet position combinations were examined, i.e., 1-1', 22', ..., 6-6'. The dimensions of $50 \mathrm{~m}$ by $20 \mathrm{~m}$ by $1.5 \mathrm{~m}$ were used in this analysis. The comparison of hydraulic measures amongst all six cases shows rather insignificant differences from Case No 2 in Table 2 , which implied that for this pond layout, the inlet/outlet position does not significantly influence the hydraulic retention time of WSPs.

\subsection{Effect of inflow directions}

A total of 5 different inflow directions were studied as shown in Figure 7, with the outflow direction normal to the outlet wall $A^{\prime} C^{\prime}$. Other conditions were the same as defined in the previous section, other than that the inlet/outlet positions correspond to $1-1$ ' in

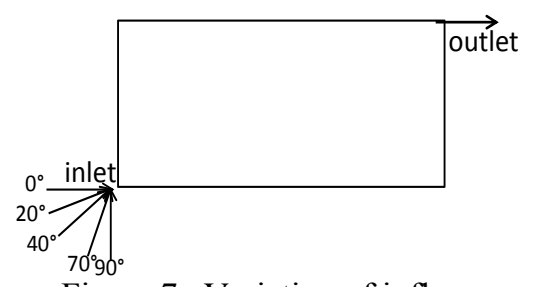

Figure 7. Variation of inflow direction

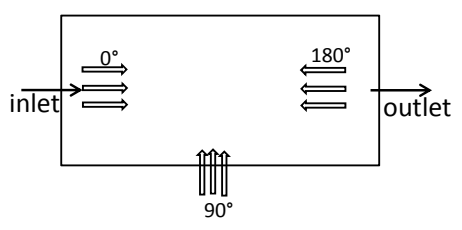

Figure 8. Variation of wind direction

Figure 1(b). Similar to the conclusion in Section 5.2, the variation of the inflow direction has an insignificant effect on WSP retention time. All measures have the same values as shown in Case No 2 in Table 2. This is due to the local boundary effect around the inlet, which dominates the effect of the inlet direction change. It can also be related to the pond configurations specified in this scenario. A further study regarding the effect of inflow direction change on WSP retention time of other pond layouts is currently in progress.

\subsection{Effect of wind speed and \\ direction}

Two wind speeds and three wind directions, resulting in a total of 6 cases were studied to investigate the wind effect on WSP retention time. The wind speed $V_{W}$ was chosen to vary from $2 \mathrm{~m} / \mathrm{s}$ to $5 \mathrm{~m} / \mathrm{s}$, blowing over the surface of the pond from $\theta_{W}=0^{\circ}, 90^{\circ}$ and $180^{\circ}$ in relation to the inflow direction (see Figure 8). These values are chosen to represent typical cases in real situations. The results of the no wind case were also presented for comparison purposes. It is easily

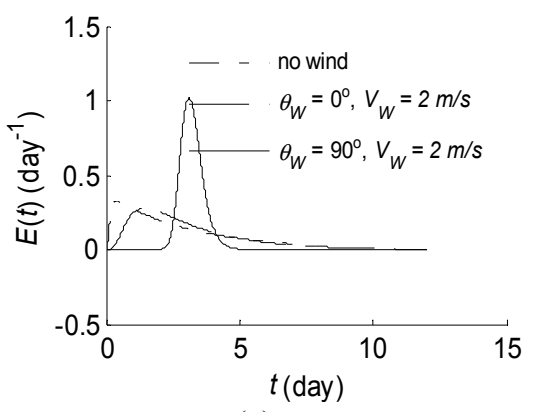

(a)

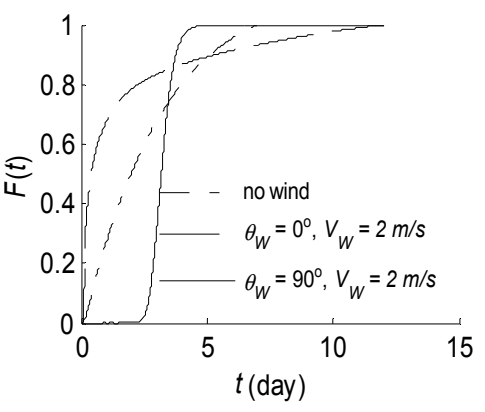

(b)
Figure 9. Tracer analysis of ponds with varying wind conditions: (a) $E(t)$ function and (b) $F(t)$ function 
identified that winds have a significant impact on WSP hydraulics particularly the wind direction relevant to the inflow direction, which has far greater effect than the wind speed. From the results shown in Figure 9 and Table 3, winds can be grouped into two categories, i.e. parallel to the inflow direction $0^{\circ}$ and $180^{\circ}$ and orthogonal to the inflow direction $90^{\circ}$. By comparing with the no wind case, it can be concluded that parallel winds have an adverse effect on WSP hydraulic efficiency by reducing the retention time, whereas orthogonal winds are more favourable. More specifically, all temporal indicators $t_{16}, t_{p}$ and $t_{m}$ are rather small

for cases 1, 2 and 5, 6 in comparison to cases 3 and 4. Parallel winds create a circulation pattern in alignment with the inlet/outlet direction, thus wastewater short circuits through the outlet and does not remain in the pond for a sufficient period of time. On the other hand, orthogonal winds develop a circulation in the transverse direction, and wastewater stays in the pond much longer to receive sufficient treatment. Overall, constructing ponds with inlet/outlet orientation in alignment with the direction of prevailing winds is not recommended.

\section{CONCLUSIONS AND FUTURE WORK}

This study investigated the hydrodynamic retention time distribution of WSPs using three-dimensional numerical models. A model convergence test was conducted and the model was validated against empirical formulations for the wind-driven circulation in a pond. The validated model was employed in a parametric analysis to identify the effects of pond configuration, inlet/outlet design and wind effects on WSP retention times. It is concluded that pond $L / W$ ratio and wind directions impose significant influence on pond performance. The greater the pond $L / W$, the longer the retention time. It is also noted that a pond should not be constructed with its inlet/outlet orientation in alignment with the prevailing wind directions to avoid reduced retention time. Further studies in terms of the interrelated effects of multiple factors on pond efficiency are in progress. In addition, the evaluation of WSP hydrodynamic efficiency will ultimately be incorporated with the biological/physicochemical/biochemical parameters to interpret the ultimate efficacy of waste stabilization ponds.

\section{REFERENCES}

Abbas, H., Nasr, R., Seif, H., 2006. Study of waste stabilization pond geometry for the wastewater treatment efficiency. Ecological Engineering 28 (1), 25-34.

Badrot-Nico, F., Guinot, V., Brissaud, F., 2010. Taking wind into account in the design of waste stabilisation ponds. Water Science and Technology 61 (4), 937.

Department of Environment and Planning (DEP), 1992, Performance Assessment of Scheduled Sewage Treatment Plants in Tasmania

Fogler, H.S., 1992. Elements of chemical reaction engineering. Prentice-Hall.

Kennedy, M., Ahlfeld, D., Schmidt, D., Tobiason, J., 2006. Three-Dimensional Modeling for Estimation of Hydraulic Retention Time in a Reservoir. Journal of Environmental Engineering 132 (9), 976-984.

Persson, J., 2000. The hydraulic performance of ponds of various layouts. Urban Water 2 (3), 243-250.

Sah, L., Rousseau, D.P.L., Hooijmans, C.M., Lens, P.N.L., 2011. 3D model for a secondary facultative pond. Ecological Modelling 222 (9), 1592-1603.

Vega, G.P., Pena, M.R., Ramirez, C., Mara, D.D., 2003. Application of CFD modelling to study the hydrodynamics of various anaerobic pond configurations. Water Science and Technology 48 (2), 163-171.

Watters, G.Z., Mangelson, K.A., George, R.L., 1973. The Hydraulics of Waste Stabilization Ponds. Utah Water Research Laboratory.

Wood, M., 1997. Development of computational fluid dynamic models for the design of waste stabilisation ponds, Department of Chemical Engineering. University of Queensland, Brisbane.

Wood, M.G., Greenfield, P.F., Howes, T., Johns, M.R., Keller, J., 1995. Computational Fluid Dynamic Modeling of Waste-Water Ponds to Improve Design. Water Science and Technology 31 (12), 111-118.

Wood, M.G., Howes, T., Keller, J., Johns, M.R., 1998. Two dimensional computational fluid dynamic models for waste stabilisation ponds. Water Research 32 (3), 958-963. 